\title{
Perfil epidemiológico de los pacientes con Covid 19 unidad de cuidados intensivos en un Hospital Nacional de la ciudad de Lima 2020.
}

Epidemiological profile of patients with Covid 19 unit of intensive care in a national Hospital of the city of Lima 2020.

\author{
Marivel Rosa Martínez Véliz ${ }^{1, a}$, Belinda Olga Garcia Inga 1,b , Jenny Giovanna Poma Salinas ${ }^{1, c}$, \\ Rosario Elena Cuadros Ríos ${ }^{1, \mathrm{~d}}$
}

\section{RESUMEN}

Objetivo: Determinar el perfil epidemiológico de los pacientes con Covid 19 unidad de cuidados intensivos en un Hospital nacional de la ciudad de Lima 2020. Material y Métodos: Estudio observacional y descriptivo, la muestra conformada por 77 pacientes atendidos en la UCI. Resultados: El 78,8\% de sexo masculino, 25,9\% tienen más de 70 años, tiempo de enfermedad antes de su hospitalización 6 a 10 días, el diagnóstico médico de ingreso neumonía por Covid con $51,2 \%$, el síntoma principal la disnea $29 \%$, el $14 \%$ con comorbilidad a la hipertensión arterial, obesidad y diabetes mellitus tipo 2 , el 78,8\% utilizaron ventilador mecánico, monitor multiparámetros y bombas, el 77,5\% usó la técnica de pronación, el 51,2\% tuvo procedimiento invasivo con tubo endotraqueal, catéter venoso central, sonda nasogástrica y Foley, el 20\% tuvo como complicación la lesión por presión de $\mathrm{II}^{\circ}$, el diagnóstico de egreso fue de Covid 19 en un 100\%, la estancia hospitalaria de 11 a 21 días, mortalidad del 62\%. Conclusiones: El perfil epidemiológico de los pacientes con Covid 19\% unidad de cuidados intensivos es amplio que afectó más a los mayores de 70 años, al sexo masculino, teniendo como comorbilidad principal a la hipertensión arterial, obesidad y diabetes mellitus tipo 2, con una alta tasa de mortalidad.

PALABRAS CLAVE: Covid 19, paciente, unidad de cuidados intensivos, perfil epidemiológico.

\section{SUMMARY}

Objective: To determine the epidemiological profile of patients with Covid 19 unit of intensive care in a national Hospital of the city of Lima 2020. Material and Methods: Observational and descriptive study, the sample made up of 77 patients treated in the ICU. Results: $78.8 \%$ of the male sex, $25.9 \%$ are over 70 years old, illness time before hospitalization 6 to 10 days, the medical diagnosis of Covid pneumonia admission with $51.2 \%$, the main symptom dyspnea $29 \%$, the $14 \%$ with comorbidity to arterial hypertension, obesity and type 2 diabetes mellitus, $78.8 \%$ used a mechanical ventilator, multiparameter monitor and pumps, $77.5 \%$ used the pronation technique, $51.2 \%$ had an invasive procedure with endotracheal tube, central venous catheter, nasogastric tube and Foley, 20\% had as a complication the pressure injury of II ${ }^{\circ}$, the discharge diagnosis was Covid 19 in $100 \%$, the hospital stay of 11 to 21 days, mortality of $62 \%$. Conclusions: the epidemiological profile of patients with Covid $19 \%$ intensive care unit is

\footnotetext{
Universidad Privada de Huancayo Franklin Roosevelt. Huancayo. Perú

Docente; Lic. Enfermería; Magister; ORCID ID 0000-0003-1992-1865

Docente; Lic. Enfermería; Magister; ORCID ID: 0000-0003-1500-5253

Docente. Lic. Enfermería; ORCID ID: 0000-0002-3325-1258

Docente; Lic. Enfermería; Magister; ORCID ID: 0000-0003-1996-1621
} 
broad, affecting more those over 70 years of age, the male sex, having as main comorbidity arterial hypertension, obesity and type 2 diabetes mellitus, with a high mortality rate.

KEYWORDS: Covid 19, patient, intensive care unit, epidemiological profile.

\section{INTRODUCCIÓN}

La investigación titulada: Perfil epidemiológico de los pacientes con Covid 19 unidad de cuidados intensivos en un Hospital Nacional de la ciudad de Lima 2020, se originó en el interés de aportar datos importantes sobre la pandemia que aqueja al mundo entero porque la Organización Mundial de la Salud (OMS) la reconoció como una pandemia global el 11 de marzo de 2020; actualmente nuestro país Perú viene enfrentando dicha pandemia con muchos casos de morbimortalidad (1).

Los síntomas informados incluyen fiebre, tos, fatiga, neumonía, dolor de cabeza, diarrea, hemoptisis y disnea. Concluyendo: Durante este período inicial, la investigación publicada exploró principalmente la epidemiología, las causas, la manifestación clínica y el diagnóstico, así como la prevención y el control del nuevo coronavirus (2).

El doctor, Li Wenliang, dijo el 31 de diciembre se había desarrollado un virus desconocido en las provincias de Wuhan y Hubei, como el SARS y el MERS; los síntomas fueron encontrados fiebre, dolor de garganta y estornudos por una mujer en el hospital. La revisión explica además que a través del estornudo y el contacto humano, el virus se propagó a toda la comunidad del mundo (3).

La incidencia del síndrome de dificultad respiratoria aguda (SDRA), shock séptico, pérdida cardíaca aguda, disfunción hepática y hemorragia gastrointestinal fue significativamente mayor en el grupo de sepsis, los pacientes con COVID-19 tienen una enfermedad más grave y un peor pronóstico después de la progresión a sepsis (4).

La edad media de los pacientes fue de 59,7 años, el $67 \%$ eran hombres, $40 \%$ tenían enfermedades crónicas, $98 \%$ tenían fiebre. El $61,5 \%$ de los pacientes habían muerto a los 28 días. La mayoría de los pacientes tenían daño en la función de órganos, incluidos $67 \%$ con SDRA, 29\% con daño renal agudo, 23\% con daño cardíaco, $29 \%$ con disfunción hepática y uno $2 \%$ con neumotórax, y $71 \%$ pacientes requirieron ventilación mecánica (5).
Los factores de riesgo detectados: adulto mayor, hipertensión arterial y obesidad; los principales síntomas, tos, fiebre y disnea; los hallazgos de laboratorio frecuentes, proteína $\mathrm{C}$ reactiva elevada y linfopenia; la presentación radiológica predominante, el infiltrado pulmonar intersticial bilateral (6).

Las características epidemiológicas predominantes fueron sexo masculino, edad de 60 a 79 años y comorbilidades (hipertensión arterial y obesidad); los principales signos y síntomas al ingreso hospitalario fueron disnea, fiebre, tos y frecuencia respiratoria aumentada (7). Durante la estancia hospitalaria, el $60,87 \%$ de los pacientes fueron atendidos en la Unidad de Cuidados Intensivos (UCI) y el 69,57 \% estuvieron en ventilación mecánica; el 39,13\% recibieron un esquema terapéutico de hidroxicloroquina más azitromicina y en el 39,13\% agregaron corticoides al tratamiento mencionado. Los pacientes fallecieron antes de los 20 días de hospitalización, al día cinco la probabilidad de sobrevida general fue del 43,48 $\%$; los pacientes fallecidos fueron, principalmente, adultos mayores de sexo masculino con enfermedades preexistentes como hipertensión arterial y obesidad y clasificados con infección COVID-19 moderada a severa al ingreso hospitalario (7).

Las manifestaciones clínicas del Covid fueron: fiebre $84 \%$, tos seca $84 \%$, disnea $56 \%$, odinofagia $56 \%$, coriza $32 \%$, cefalea $24 \%$, dolor torácico $24 \%$, diarrea $16 \%$, mialgia $8 \%$, y fatiga $4 \%$ (8). Las comorbilidades el 16\% (hipertensión arterial, diabetes mellitus tipo 2, cáncer, asma e hipotiroidismo). Requirió hospitalización el 40\% (10/25) de los casos, y sólo $8 \%(2 / 25)$ necesitó ser admitido a la Unidad de Cuidados Intensivos (8).

\section{MATERIAL Y MÉTODOS}

El método general de la investigación es el científico, el método específico es el método descriptivo. El tipo de investigación estudio observacional, descriptivo y básico, el nivel de investigación es el descriptivo.

El diseño de la investigación es descriptivo simple. El esquema es el siguiente:

M - - - O 
Dónde: M: Muestra (pacientes de la UCI de un hospital de Lima)

O: Información relevante de la variable.

La población estuvo conformado por 77 pacientes de la unidad de cuidados intensivos en un hospital nacional de la ciudad de Lima.

Muestra censal conformada por los 77 pacientes de la unidad de cuidados intensivos de un hospital nacional de la ciudad de Lima, muestreo no probabilístico intencional.

La técnica de investigación observación, el instrumento ficha de observación, con una confiabilidad de 0,798 .

\section{RESULTADOS}

En la tabla 1 y el gráfico 1 se observa que de $100 \%$ pacientes con COVID 19 atendidos en la unidad de cuidados intensivos en un Hospital Nacional de la ciudad de Lima, 20(25,9\%) eran más de 70 años, 78,8\% son de sexo masculino, $22,5 \%$ procedieron de Covid1, $52,5 \%$ el tiempo de enfermedad en casa entre 6 a 10 días, $51,2 \%$ tuvieron diagnóstico médico de ingreso a neumonía por covid, $29,1 \%$ presentaron como síntoma principal la disnea, $14 \%$ con comorbilidad a HTA, obesidad y DM tipo $2,78,8 \%$ los equipos que utilizaron fue VM, monitor y bombas infusoras, $77,5 \%$ hicieron uso de la pronación, $51,2 \%$ tuvieron procedimientos invasivos con TET, CVC, SNG, SF,

Tabla 1. Perfil epidemiológico de los pacientes con Covid 19 unidad de cuidados intensivos en un Hospital Nacional de la ciudad de Lima 2020.

\begin{tabular}{lcc}
\hline \multicolumn{1}{c}{ Categorías } & Frecuencia & $\mathbf{\%}$ \\
\hline Edad: más de 70 años & 20 & 25,9 \\
Sexo: Masculino & 63 & 78,8 \\
Procedencia: Covid 1 & 18 & 22,5 \\
Tiempo de enfermedad antes de su hospitalización: 6 a 10 días & 42 & 52,5 \\
Diagnóstico médico de ingreso: neumonía por Covid & 41 & 51,2 \\
Síntoma de inicio: disnea & 59 & 29,1 \\
Comorbilidad: HTA, obesidad, DM tipo 2 & 11 & 14.00 \\
Equipos que utilizo: VM, monitor, bombas infusoras & 63 & 78,8 \\
Uso de pronación: si & 62 & 77,5 \\
Procedimientos invasivos: TET, CVC,SNG,SF & 41 & 51,2 \\
Complicaciones: Ninguna & 38 & 47,5 \\
Diagnóstico de egreso: Covid 19 & 77 & 100.0 \\
Estancia hospitalaria: 11 a 21 días & 36 & 45,0 \\
Condición de alta: fallecidos & 50 & 62,7 \\
\hline
\end{tabular}

Fuente: Ficha de recolección de datos

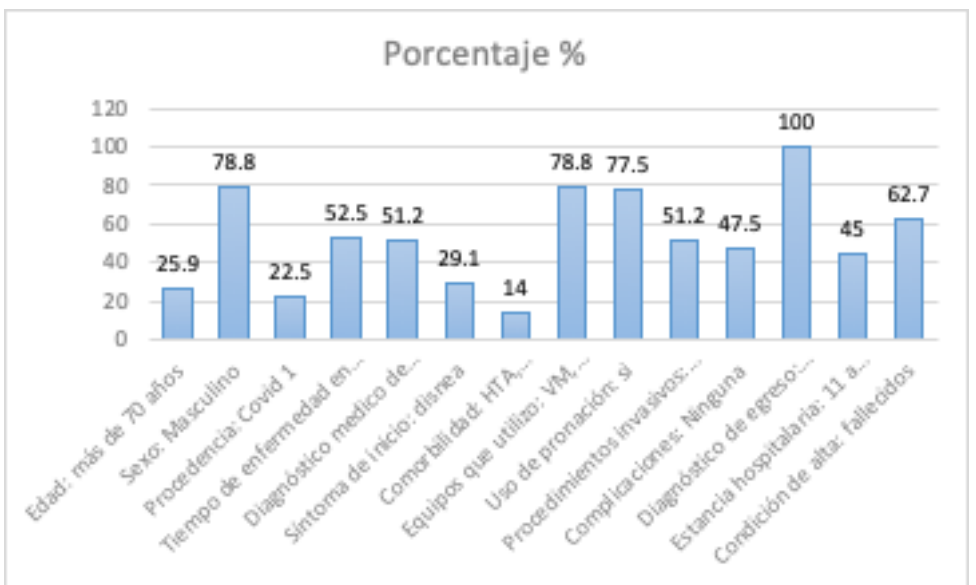

Gráfico 1. Perfil epidemiológico de los pacientes con Covid 19 unidad de cuidados intensivos en un Hospital Nacional de la ciudad de Lima 2020. 
$47,5 \%$ no presentaron ninguna complicación, $100 \%$ con diagnóstico de egreso de Covid 19, 45\% tuvieron una estancia hospitalaria entre 11 a 21 días, $62,7 \%$ su condición de egreso ha sido fallecidos.

\section{DISCUSIÓN}

Según los resultados mostrados en la tabla 1, el diagnóstico médico de ingreso neumonía por Covid con $51,2 \%$, el síntoma principal la disnea con un $29,1 \%$, con comorbilidad principal a la hipertensión arterial, obesidad y diabetes mellitus tipo 2 con un $14 \%$, los equipos que se utilizaron fueron ventilador mecánico, monitor multiparámetros y bombas infusoras en un $78,8 \%$, hicieron uso de la técnica de pronación en un $77,5 \%$, los procedimientos invasivos fueron la intubación endotraqueal, catéter venoso central, sonda nasogástrica y sonda Foley con 51,2 con ninguna complicación en un 47,5\%, el diagnóstico de egreso fue de Covid 19 en un $100 \%$, la estancia hospitalaria de 11 a 21 días en un $45 \%$, con una mortalidad del $62 \%$.

Caso similar encontraron Yang Yang, et al., en pacientes con neumonía por SARS-CoV-2 que ingresaron en la unidad de cuidados intensivos (UCI) del hospital Wuhan Jin Yin-tan (Wuhan, China), en los que de la edad de 59,7 años, $67 \%$ eran hombres, 40\% tenían enfermedades crónicas, $98 \%$ tenían fiebre, y $61,5 \%$ pacientes habían muerto a los 28 días (5).

De igual manera Acosta et al ., concluyeron que los factores de riesgo detectados fueron adulto mayor, tener hipertensión arterial y obesidad; los principales síntomas, tos, fiebre y disnea (6).

Así mismo otro estudio similar de Llaro et al., El mayor tiempo de sobrevida se observó entre los que estuvieron en ventilación mecánica. Un porcentaje alto de fallecidos $62,7 \%$ del total de pacientes atendidos pacientes atendidos en la unidad de cuidados intensivos en un Hospital Nacional de la ciudad de Lima (7).

De igual manera se coincide con Murrugarra quien concluyó que la edad como factor sociodemográfico, la presencia de factores de riesgo y el estado de salud como factores clínicos, se relacionan significativamente con la mortalidad del COVID-19 pacientes atendidos entre marzo a julio del 2020 en el Hospital II EsSalud Chocope (9). Se confirma que la mayor cantidad de pacientes atendidos fueron de sexo masculino similar a nuestro trabajo de investigación; así mismo la comorbilidad (HTA, obesidad, DM tipo
2) en el presente trabajo de investigación es de $14 \%$ del total de pacientes atendidos en la unidad de cuidados intensivos en un Hospital Nacional de la ciudad de Lima.

Así también se coincide con Escobar et al., donde el 78,6\% eran varones, edad promedio 73,4 años, factores de riesgo 92,9\% (ancianos, hipertensión y obesidad), los síntomas más frecuentes fueron disnea, fiebre y tos (10).

Se difiere con Guzmán, quien concluye que no hubo diferencias en el sexo, mayor frecuencia entre la cuarta y quinta década de la vida, cuadro clínico característico (fiebre, tos y disnea) (8).

También se difiere con Sánchez, quien halló que el $71,7 \%$ son mujeres, con una edad media de 41 años, los síntomas más frecuentes son: dolor de garganta $(60,9 \%)$, cefalea $(58,7 \%)$ y tos $(55,8 \%)$. Además, cerca del 95,6 \% de pacientes ha presentado clínica leve (11).

Según la Organización Mundial de la salud (OMS), las características epidemiológicas es cualquier característica o circunstancia detectable en una persona o grupo de personas, que se asocia con un aumento de riesgo de padecer, desarrollar o estar expuesto a un proceso mórbido (12).

Por otro lado, Sanchez, manifiesta que: Las características epidemiológicas es cualquier actividad o circunstancia detectable en una persona o grupo de personas, que se asocia con un aumento de riesgo de padecer, desarrollar o estar expuesto a un proceso mórbido (13).

Con respecto a la edad se observa que el $25,9 \%$ tienen más de 70 años, $23,8 \%$ tienen de 50 a 59 años, $22,5 \%$ de 40 a 49 años, $18,8 \%$ de 30 a 39 años, $6,3 \%$ de 60 a 69 años, 3,8\% de 20 a 29 años; estos resultados son similares al estudio realizado por Acosta $G$ et al . donde la edad promedio de 53,5 años (rango de 25 a 94)(6), de igual manera se tiene a Llaro et al.,que la edad fue de 60 a 79(7), así también es similar al estudio realizado por Murrugarra eran de 65 años 51,9\% (9), por otro lado, se coincide con Escobar et al., la edad promedio 73,4 años (rango 26 a 97)(10). Pero se difiere con Guzmán en que la edad fue de 38 años (rango: 1167 años)(8), de igual manera se difiere con el estudio de Yang, en que la edad promedio fue de 59,7años (5), finalmente diferimos con Sánchez, en el cual la edad media fue de 41 años (11). Según el Centro para el 
control y la prevención de enfermedades de España que el riesgo más alto de enfermarse gravemente a causa del COVID-19 lo tienen las personas de 85 años de edad o más (14).

En relación con el sexo de los pacientes con Covid 19 unidad de cuidados Intensivos en un Hospital Nacional de la ciudad de Lima 2020 78,8\% corresponden al sexo masculino y $17,5 \%$ corresponden al sexo femenino coincidimos con Chen refiere que el $51,3 \%$ eran hombres, de igual manera con Escobar et al., concluyen $78,6 \%$ eran varones (10), así también Acosta et al. el $76 \%$ eran varones (6), igual Yang et al., que el $67 \%$ eran hombres (7)(5), otro similar es de Murrugarra en que el sexo masculino fue de 60,4\% (9). Pero diferimos con Sánchez el 71,7\% son mujeres, de igual manera se difiere con Guzmán quien indica que el $48 \%$ fueron varones $(10,12)$. Según el Instituto da salud San Carlos. Ministerio de Ciencia e innovación España en relación con el sexo la Covid 19 afecta más a los hombres que a las mujeres (15).

De los pacientes con Covid 19 en la Unidad de cuidados Intensivos en un Hospital Nacional de la ciudad de Lima 2020, el 22,5\% proceden de Covid 1, $16,3 \%$ proceden de Covid 2, 8,8\% proceden de Covid3, $17,5 \%$ proceden de Covid 4, 16(20\%) proceden de Emergencia/Covid, 5\% proceden de SOP/Covid, 3,8\% proceden de Recuperación/Covid y 2,5\% proceden de otros servicios. Se difiere con Acosta donde el 41,2\% fueron referidos de otros establecimientos de salud (6). Según la OPS la capacidad de respuesta de un hospital puede ser superada por la llegada masiva de pacientes que soliciten atención médica como consecuencia de una situación de emergencia (16).

En relación con tiempo de enfermedad antes de su hospitalización de los pacientes con Covid 19 en la Unidad de cuidados Intensivos en un Hospital Nacional de la ciudad de Lima 2020, 54,5\% tienen de 6 a 10 días de enfermedad, el 26\% tienen menos de 5 días de enfermedad antes de su hospitalización, $13 \%$ tienen de 11 a 15 días de enfermedad y $6,5 \%$ tienen más de 15 días de enfermedad. No se cuenta con antecedentes para la discusión. Según Melguizo et al., las creencias sobre el cuidado de la salud pueden considerarse desde las ideas que las personas aceptan y expresan sobre cómo conservan o recuperan su salud o como conductas verbales que las personas expresan (17).

Del dagnóstico médico de ingreso de los pacientes con Covid 19 el 51,2\% ingresaron con diagnostico medico de neumonía por COVID, 43,8\% ingresaron con diagnóstico médico IRA por Covid, y 1,3\% ingresaron con diagnóstico médico falla respiratoria. A diferencia de Chen et al., en su estudio refiere que el $18,2 \%$ estaban en el grupo de sepsis y el $81,8 \%$ sin sepsis (4), así también Yang el total de sus pacientes críticos eran con neumonía por SARSCoV-2 (5). La COVID-19 es un virus de la gran familia de los coronavirus, un tipo de virus que infecta a seres humanos y algunos animales, causando una enfermedad respiratoria llamada enfermedad por coronavirus de 2019 Covid 19.

Con respecto a los síntomas de inicio de la enfermedad de los pacientes con Covid 19 unidad de cuidados Intensivos en un Hospital Nacional de la ciudad de Lima 2020, se han obtenido 203 síntomas de los cuales, $529,1 \%$ presentaron disnea, $23,6 \%$ presentaron tos, $18,7 \%$ presentaron fiebre, $11,8 \%$ presentaron malestar general, 5,9\% presentaron cefalea, 3,9\% presentaron dolor torácico, 3,4\% presentaron dolor de garganta, $1,9 \%$ presentaron erupcionó cutánea, $0.9 \%$ otros. Coincidimos con Acosta mencionando que los principales síntomas son tos, fiebre y disnea, caso similar es el de Guzmán donde las manifestaciones clínicas fueron: fiebre $84 \%$, tos seca $84 \%$, disnea $56 \%$, odinofagia $56 \%$, coriza $32 \%$, cefalea $24 \%$, dolor torácico $24 \%$, diarrea $16 \%$, mialgia $8 \%$, y fatiga $4 \%$ (8), y se difiere con Yang quien halló que los pacientes de su estudio tenían fiebre 61,5\% (5). Pero se difiere parcialmente con Sánchez quien indica : dolor de garganta $60,9 \%$, cefalea $58,7 \%$ y tos $55,8 \%$. Según la OMS los signos y síntomas del Covid 19 son: fiebre, tos seca, cansancio; otros síntomas menos frecuentes: pérdida del gusto o el olfato, congestión nasal, conjuntivitis, dolor de garganta, dolor de cabeza, dolores musculares o articulares, diferentes tipos de erupciones cutáneas, náuseas o vómitos, diarrea, escalofríos o vértigo(11). Los síntomas del cuadro grave de la COVID-19 son: disnea, pérdida de apetito, confusión, dolor en el pecho, temperatura alta. Otros síntomas menos frecuentes: irritabilidad, convulsiones, ansiedad, depresión, trastornos del sueño, complicaciones neurológicas más graves y raras, accidentes cerebrovasculares, inflamación del cerebro, estado delirante y lesiones neurales.

Con respecto a la comorbilidad prexistente de los pacientes con Covid 19 unidad de cuidados Intensivos en un Hospital Nacional de la ciudad de Lima 2020, el $36 \%$ no tenían ninguna comorbilidad, $14 \%$ tenían como comorbilidad a HTA más obesidad más DM tipo 2, 9\% tenían como comorbilidad a obesidad, 6\% tenían como comorbilidad a la HTA, 6\% tenían 
como comorbilidad a DM tipo 2, 5\% tenían como comorbilidad a DM tipo 2 más obesidad, $1 \%$ tenían como comorbilidad enfermedad renal y asma, , $1 \%$ tenían como comorbilidad a neumotórax y falla renal, $4 \%$ tenían como comorbilidad a la obesidad mórbida, 5\% tenían como comorbilidad a PO de cesárea, preclampsia, síndrome de Hellp y puérpera, 1\% tenían como comorbilidad a PO colecistectomía más Obesidad mórbida, PO craneotomía descomprensiva por hematoma, $1 \%$ tenían como comorbilidad a síndrome convulsivo más quemadura $\mathrm{II}^{\circ}, 6 \%$ tenían como comorbilidad otros como hipotiroidismo, asma, hipoalbuminemia, TBC, cardiopatía, lo que coincide con Acosta quien concluye que los factores de riesgo fueron hipertensión arterial y obesidad (6), así tambien Guzmán el 16\% las comorbilidades fueron hipertensión arterial, diabetes mellitus tipo 2, cáncer, asma e hipotiroidismo(8), y se coincide parcialmente con Sánchez fueron la obesidad, el asma y la hipertensión arterial (11), finalmente se coincide parcialmente con Escobar et al., indicando el $92,9 \%$ a la hipertensión y obesidad (10). Se difiere con Yang quien señala que $40 \%$ tenían enfermedades crónicas (5). Según el INEI las comorbilidades a Covid 19, son la hipertensión arterial, las enfermedades cardiovasculares graves, el cáncer, la diabetes mellitus (18).

Con respecto a los equipos que utilizaron durante la hospitalización de los pacientes con Covid 19: En el $78,8 \%$ se utilizaron VM, bombas infusoras y $5 \%$ utilizaron monitor, bombas infusoras, cánulas de alto flujo, 12,5\% utilizaron VM, monitor, bombas infusoras, hemodializador, similar al estudio de Acosta donde el 41,2\% ingresó a ventilación mecánica (6), así también se coincide con Llaro et al., el 69,57\% estuvieron en ventilación mecánica (7), similar también al estudio realizado por Escobar et al., 6\% ingresaron a ventilación mecánica (10). Según la norma técnica de la unidad de cuidados intensivos los equipos que se requieren para brindar atención a los pacientes son: ventilador mecánico, bombas de infusión, oxigenoterapia, sistemas de aspiración, monitor, desfibrilador, coche de paro, cestos, pulsioxímetros, electrocardiógrafos, laringoscopio, etc.

Con respecto al uso de la técnica de pronación en el tratamiento de los pacientes con Covid 19 unidad de cuidados Intensivos en un Hospital Nacional de la ciudad de Lima 2020, se observa que $80,5 \%$ hicieron uso de la técnica de pronación y $19,5 \%$ no hicieron uso de la técnica de pronación. Según Díaz, el decúbito prono (DP) se ha utilizado como opción de tratamiento en pacientes con Síndrome de Distrés Respiratorio Agudo (SDRA), su uso permite aumentar el volumen final de espiración del pulmón y un mayor reclutamiento alveolar, mejorando la oxigenación en pacientes con insuficiencia respiratoria severa (19). Con respecto a los procedimientos invasivos realizados a los pacientes con Covid 19 en la Unidad de cuidados Intensivos en un Hospital Nacional de la ciudad de Lima 2020, $51,2 \%$ se les realizó procedimientos invasivos con, TET, CVC,SNG, SF, 30\% se les realizó procedimientos invasivos con TET, CVC,SNG, 8,8\% se les realizó procedimientos invasivos con TET, CVC,SNG, SF, línea arterial, CAF , SF, línea arterial, $2,5 \%$ se les realizó procedimientos invasivos con TET, CVC,SNG, SF, línea arterial, CAF, drenaje toráxico, TQT, $3,8 \%$ se les realizó procedimientos invasivos con TET, CVC,SNG, SF, línea arterial, CAF, drenaje toráxico. No se tiene antecedentes para la discusión y de acuerdo a la norma técnica de la UCI son procedimientos recomendados para tratar al paciente crítico.

Con respecto a las complicaciones durante la hospitalización de los pacientes con Covid 19 de la Unidad de cuidados Intensivos en un Hospital Nacional de la ciudad de Lima 2020 se observa que $47,5 \%$ no tuvieron ninguna complicación pero el $20 \%$ tuvieron como complicación lesión por presión $\mathrm{II}^{\circ}, 8,8 \%$ tuvieron como complicación insuficiencia renal/falla renal, $8,8 \%$ tuvieron como complicación falla multiorgánica, $5 \%$ tuvieron como complicación neuropatía, 6,3\% tuvieron como complicación neumonía intrahospitalaria. Se difiere con Yang que indica 29\% tuvieron daño renal agudo y en mismo porcentaje tuvieron disfunción hepática (5). Según la Organización Panamericana de la Salud (OPS) hay complicaciones con el aparato respiratorio, neurológicas, delirio o encefalopatía, accidente cerebrovascular, meningoencefalitis, alteración de los sentidos del olfato (anosmia) y el gusto (disgeusia), ansiedad, depresión y problemas del sueño (12). En muchos casos las manifestaciones neurológicas se han reportado incluso en ausencia de síntomas respiratorios. También hay reportes de casos de síndrome de Guillain Barré en pacientes con COVID-19.

En lo que respecta al diagnóstico de egreso de los pacientes con Covid 19 en la Unidad de cuidados Intensivos en un Hospital Nacional de la ciudad de Lima el $100 \%$ tuvieron como diagnóstico de egreso COVID-19, similar se tiene a Escobar et al., quienes concluyen que los fallecidos por COVID-19 presentaron neumonía severa bilateral (10). 
Con respecto a la estancia hospitalaria de los pacientes con Covid 19 en la Unidad de cuidados Intensivos en un Hospital Nacional de la ciudad de Lima 2020, el $45 \%$ estuvieron hospitalizados 11 a 21 días, $25 \%$ menos de 10 días, $13,8 \%$ entre 22 a 30 dias y $12,5 \%$ más de 31 días. Se difiere con Yang quien refiere que $61,5 \%$ pacientes murieron a los 28 días y de 7 días para los sobrevivientes (5). Según Anyarin la estancia hospitalaria se define como una unidad de medida de permanencia del paciente en régimen de hospitalización ocupando una cama en un intervalo de tiempo y se calcula por la diferencia entre la fecha de alta y la de ingreso, es el número total de días que permanece hospitalizado un paciente desde su ingreso hasta el día de alta del hospital (20).

Con respecto a la condición de alta de los pacientes con Covid 19 en la Unidad de cuidados Intensivos en un Hospital Nacional de la ciudad de Lima 2020, se observa que $35,1 \%$ fueron recuperados y $64,9 \%$ fueron fallecidos; se difiere con Acosta indicando que el 29,4\% falleció, de igual manera se tiene a Guzmán que no tuvo ningún fallecido y finalmente se tiene a Chen quien refiere la mortalidad hospitalaria general fue del 3,3\% (4,6,10).

\section{Correspondencia:}

Marivel Rosa Martínez Véliz

Av. Giráldez No 542 .Huancayo, Perú

\section{REFERENCIAS BIBLIOGRAFICAS}

1. Organización Mundial de la Salud. Información basíca sobre la COVID-19.Ginebra: Organización Mundial de la Salud; Octubre 2020. (Citado el 18 de marzo del 2021) Disponible en: https://www.who.int/ es/news-room/q-a-detail/coronavirus-diseasecovid-19

2. Adhikari S, Meng S, Wu YJ, et al. Epidemiology, causes, clinical manifestation and diagnosis, prevention and control of coronavirus disease (COVID-19) during the early outbreak period: a scoping review. Infect Dis Poverty.2020;9: 29. DOI: 10.1186/s40249-020-00646-x

3. Khan N, Naushad M. Effects of Corona Virus on the World Community. SSRN. 2020. DOI: 10.2139/ ssrn. 3532001

4. Chen Q, Shao J. Risk Factors of Coronavirus Disease 2019 Patients Progressing to Sepsis. SSRN. 2020. (Citado el 18 de marzo del 2021) Disponible en: https://ssrn.com/abstract=3576956

5. Yang X, Yu Y, Xu J, et al. Clinical course and outcomes of critically ill patients with SARS-CoV-2 pneumonia in Wuhan, China: a single-centered, retrospective, observational study. Lancet Respir Med. 2020 May; 8(5): 475-481.doi: 10.1016/S2213-2600(20)30079-5

6. Acosta G, Escobar G, Bernaola G, et al. Caracterización de pacientes con COVID-19 grave atendidos en un hospital de referencia nacional del Perú. Rev. perú. med. exp. salud publica. 2020;37(2):253-258. $\quad$ DOI: $\quad 10.17843 /$ rpmesp.2020.372.5437

7. Llaro M, Gamarra B. Campos K E. Características clínico-epidemiológicas y análisis de sobrevida en fallecidos por COVID-19 atendidos en establecimientos de la Red Sabogal-Callao 2020. Horizonte Médico. 2020; 20(2): e1229. DOI: 10.24265/horizmed.2020.v20n2.03

8. Guzmán O, Lucchesi-Vásquez EP, TrellesDe Belaúnde $\mathrm{M}$, et al.. Características clínicas y epidemiológicas de 25 casos de COVID-19 atendidos en la Clínica Delgado de Lima 2020. Revista De La Sociedad Peruana De Medicina Interna. 2020; 33(1): 15-24. DOI: 10.36393/spmi.v33i1.506

9. Murrugarra S. Factores sociodemográficos y clínicos relacionados a la mortalidad en pacientes COVID-19. Hospital II EsSalud Chocope Trujillo 2020.Tesis de maestría. Trujillo: Universidad César Vallejo; 2020. (Citado el 18 de marzo del 2021) Disponible en: https://repositorio.ucv.edu.pe/ bitstream/handle/20.500.12692/49032/Murrugarra SST\%20-\%20SD.pdf? sequence=1\&isAllowed=y.

10. Escobar G, Mata J, Taype W, Ayala R, Amado José. Características clínico-epidemiológicas de pacientes fallecidos por COVID-19 en un hospital nacional de Lima, Perú. Revista de la Facultad de medicina Universidad Ricardo Palma.2020; 20(2).

11. Sánchez C. Características clínicas y epidemiológicas de personal sanitario con COVID- 19 del primer nivel de atención de Lima Norte, Abril - Junio, 2020. Tesis de Grado. Trujillo: Universidad Privada Anternor Orrego; 2020. (Citado el 18 de marzo del 2021) Disponible en: https://repositorio.upao.edu.pe/ handle/20.500.12759/6620

12. Organización Panamericana de la Salud. Instructivo - Lista de verificación de alistamiento para la respuesta al COVID-19 en Hospitales - OPS/OMS. Washington DC:| Organización Panamericana de la Salud; 2020. (Citado el 18 de marzo del 2021) Disponible en: https://www.paho.org/es/documentos/ instructivo-lista-verificacion-alistamiento-pararespuesta-al-covid-19-hospitales

13. Sanchez A. Características clínicas y epidemiológicas de personal sanitario con Covid- 19 del primer nivel de atención de Lima Norte, Abril - Junio, 2020. Tesis de Grado. Trujillo: Universidad Privada Anternor Orrego; 2020.

14. Centro para el controly la prevención de enfermedades. Cómo protegerse y proteger a los demás Madrid: Centro para el controly la prevención de enfermedades; 
2020 (Citado el 18 de marzo del 2021) Disponible en: https://espanol.cdc.gov/coronavirus/2019-ncov/ your-health/need-to-know.html

15. Instituto da salud San Carlos. ¿Qué factores de riesgo hay en el desarrollo de la COVID-19? Madrid: Ministerio de Ciencia e innovación;2020. (Citado el 18 demarzodel2021)Disponibleen:https://www.isciii.es/ InformacionCiudadanos/DivulgacionCultura Cientifica/DivulgacionISCIII/Paginas/Divulgacion/ FactoresPronosticosCoronavirusDivulgacion.aspx

16. Organización Panamericana de la Salud. Coronavirus. Washington DC: Organización Panamericana de la Salud; 2020. (Citado el 18 de marzo del 2021) Disponible en: https://www.paho.org/es/temascorona virus

17. Melguizo E,y Alzate M. Creencias y prácticas en el cuidado de la salud.| Avances en Enfermería.2008; 26(1); 11-123. (Citado el 18 de marzo del 2021) Disponible en: https://revistas.unal.edu.co/index.php/ avenferm/article/view/12891/13649

18. Instituto Nacional de Estadística e Informática. Factores de riesgo asociados a complicaciones por Covid 19. Lima: Instituto Nacional de Estadística e Informática; Junio 2020. (Citado el 18 de marzo del 2021) Disponible en: https:/www.inei.gob.pe/ media/MenuRecursivo/publicaciones_digitales/Est/ Lib1738/libro.pdf.

19. Díaz N. Población y Muestra. Ciudad de Mexico: Universidad Autónoma del Estado de Mexico; 2016. (Citado el 18 de marzo del 2021) Disponible en: https:/core.ac.uk/download/pdf/80531608.pdf

20. Anyarin Y. Factores que influyen en la estancia prolongada en el servicio de medicina del hospital Félix Torrealva Gutiérrez. ICA -2016. Tesis de Gardo. Arqeuipa, Perú: Universidad Nacional de San Agustín de Arequipa; 2017. (Citado el 18 de marzo del 2021) Disponible en: http://repositorio. unsa.edu.pe/bitstream/handle/UNS A/6323/ ENManpiyy.pdf? sequence $=1 \&$ is Allowed $=y \#: \sim$ :text=LA $\% 20$ ESTANCIA $\% 20$ HOSPITALARIA,$\mathrm{Se} \% 20$ define $\% 20$ como\&text $=\mathrm{Se} \% 20$ define $\% 20$ tambi\%C3\%A9n\%20como\%20el,d\%C3\%ADa\%20 de $\% 20$ alta $\% 20$ del $\% 20$ hospital

Recibido: 27/03/2021

Aceptado: 15/05/2021 